\title{
Comparative Investigation of the Sensitivity of Candida Fungi Isolated From Vulvovaginal Candidiasis to Nystatin and Teucrium polium Smoke Product
}

\author{
Marzieh Akbarzdeh $^{1 \mathbb{D}}$, Batool Bonyadpour ${ }^{*}{ }^{\mathbb{D}}$, Kayvan Pakshir $^{3}$, Abdol Ali Mohagheghzadeh ${ }^{4}$
}

\begin{abstract}
Objectives: The present study aimed to compare the antifungal activities of Teucrium Polium smoke product and nystatin in the treatment of Candida vaginitis in vitro.

Materials and Methods: In this quasi-experimental study, 105 subjects were diagnosed with Candida vaginitis. The data were collected through a collection form and the species were isolated by the germ tube, as well as CHROMagar chromogenic and chlamydospore formation tests.

Results: Based on the results of the germ tube, chlamydospore formation, and CHROMagar tests, $70.5 \%, 23.8 \%$, and $66.6 \%$ of the species were Candida albicans, respectively. In addition, $99 \%$ of the samples were sensitive to nystatin. A significant relationship was also observed between the antifungal drug and the type of organism $(P<0.02)$. Finally, all the 15 standard clinical samples were sensitive to T. polium smoke.

Conclusions: In general, the results confirmed the antifungal effects of T. polium and nystatin on the species isolated from 10 clinical samples obtained from Candida vaginitis as well as on five standard strains.

Keywords: Vulvovaginal candidiasis, Albicans, Nystatin, Teucrium Polium, Smoke, Persian medicine
\end{abstract}

\section{Introduction}

Vaginal candidiasis infection (VVC) is a common problem among women and requires consultation and reference to primary healthcare centers (1). In addition, VVC infects the gastrointestinal tract and vagina by some Candida species. Candida albicans is reported as the cause of $85 \%$ $95 \%$ of the cases and Candida glabrata is mentioned as the most prevalent cause of non-albicans Candida vaginitis (2). Overall, in one study, the incidence of Candida infection was estimated as eight per 100000 population (3), thus requiring adherence to epithelial cells. Based on some previous reports, C. albicans have the highest rate of adherence $(4,5)$. A previous epidemiological study on the Candida species leading to VVC in Iran revealed that $C$. albicans (67\%) was responsible for this infection, followed by Teucrium polium (18.3\%), and C. glabrata (6. 8\%). Albicans and glabrata species were also dominant among the infections involving several species simultaneously (6). Therefore, VVC mostly results from C. albicans. However, the attacks of non-albicans Candida species are increasing nowadays. Most non-albicans Candida species have greater resistance to the medicines which contain azoles and their resultant infection is difficult to treat as well. Hence, physicians normally perform the treatment experimentally instead of routine laboratory techniques (7).

Although VVC is not life-threatening, it may cause complications for the patients and lead to their waste of time and money. Moreover, VVC is a psychosomatic disorder which results in stress in the patients, particularly its recurrent form (8). So far, the most common medicines for Candida include nystatin (polyene), clotrimazole, miconazole, ketoconazole (imidazole), and triazole which might have various side effects. In addition, considering their wide spectrum, they could have different sensibilities (9-12). Given the sensitivity of Candida isolates to antifungal drugs, particularly the azoles, as well as the side effects of nystatin, more attention is now paid to biologically active compounds which are obtained from the plants that were used in herbal medicine (13). Over the past decade, the use of complementary and alternative medicine has increased for treating female problems such as menopause, premenstrual syndrome, as well as sexual and menstrual problems and virginities (14-21).

Teucrium polium is regarded as one of the most important members of the Teucrium family which is 
abundantly found in South-Western Asia, Europe, the North of Africa, and the South and Northeast of Iran. Twelve species occur in Iran including three endemic species (22). The plant has 220 genera and almost 4000 species worldwide (23).

Further, T. polium in folk medicine and traditional medicinal plant is used for numerous ailments in Iran (24). For example, Khoshnood-Mansoorkhani et al reported that 28 compounds were identified in the essential oil of this plant with $99.75 \%$, in addition to the combination of a-pinene (12.52\%), linalool (10.63\%), caryophyllene oxide $(6.69 \%), \beta$-pinene $(7.09 \%)$, and caryophyllene (6.98\%) with $46.91 \%$ constitute the highest percentages of essential oil (25).

Due to its antimicrobial and antioxidant properties, this herb is also traditionally utilized to treat fungal in Iran and some other countries (26). Other studies further indicated that T. polium has diuretic, antipyretic, diaphoretic, anti-spasm, tonic, anti-inflammatory, antihypertensive, appetizing, antibacterial, analgesic, and anti-glucose functions (27) and its extract demonstrates antihypertensive, anti-spasm, antibacterial, antipyretic, antimicrobial, and antifungal activities. Various studies have so far focused on the properties of T. polium and evaluated the plant extract which was used as an oil, essence, aqueous extract, volatile oil, decoction, and brewed (28). The chemical analysis of T. polium indicates that this plant contains various compounds such as iridoids, flavonoid, and cirziliol (29). Furthermore, T. polium is reported to include beta-sitosterol, campesterol, csterol, glucose, fructose, raffinose, rhamnose, limonene, linalool, cedrol, and alpha-phellandrene compounds (30). However, to the best of our knowledge, no study has investigated the effect of T. polium smoke on fungi in Iran. The present study sought to compare the sensitivity of $C$. albicans species to nystatin and T. polium smoke product 48 hours after culturing.

\section{Materials and Methods}

The laboratory-experimental study was performed in the clinics affiliated to Shiraz University of Medical Sciences, Shiraz, Iran during 2007-2008. Using a randomized complete block design, the sample size was considered as 100 subjects. Thus, almost 1100 patients were evaluated for seven months, who referred to gynecology clinics of the selected hospitals in Shiraz. Among these patients, 450 ones had complaints related to genital infections out of whom, 280 patients were selected based on the history and characteristics of VVC. Finally, 105 subjects were diagnosed with VVC. Then, written informed consent was obtained for the isolation of Candida yeasts from all subjects.

In the first group, species were isolated from 15 clinical Candida samples in this study. Moreover, five Candida strains were examined, including C. albicans, C. glabrata, C. tropicalis, C. krusei, and C. parapsilosis that were approved by the Mycology Group of Medical School for Training and Research, with T. polium smoke product in vitro.

In the second groups, species were isolated from 15 clinical Candida samples, as well as five Candida strains, confirmed by the Mycology Group with antifungal nystatin, were inoculated in the laboratory. The patients with Candida infection by three tests were identified by germ tube test, CHROMagar chromomeric medium, and chlamydospore formation test in gynecological clinics. At the end of incubation, the diameter of the inhibition zone around the disk drug nystatin and T. polium were compared as well.

\section{Germ Tube Test}

The following steps were taken to perform the test of the germ tube, which is also referred to as the "Raynaud's phenomenon":

1. Organism passage for 24-48-hours;

2. Preparing human serum at $1 \mathrm{cc}$ for each sample;

3. Removing a sterile ointment as much as a loop from the organism;

4. Introducing the organism into $1 \mathrm{cc}$ of human serum;

5. Keeping the serum containing the organism at $37^{\circ}$ incubator for 2-3 hours;

6. Taking one anus from a serum suspension of 30 lambdas, followed by placing a drop of each suspension on the labeled microscope slides to examine the germ tubes.

The gland tube vision was considered in favor of $C$. albicans. A germ tube test is a screening test which for $t$ is an outgrowth which is produced by the spores of spore-releasing fungi during germination. Additionally, the germ tube test is regarded as the gold standard for the diagnosis of C. albicans. In this method, the intended yeast is inserted into human or rabbit serum diluted with normal saline $(1 / 2)$ and is then kept in the incubator at $37^{\circ} \mathrm{C}$ for a few hours. Approximately most of the isolated Candida albicans develop the germ tubes when incubated in a proteinaceous medium. The observation of the germ tube is in favor of C. albicans $(31,32)$

\section{Chlamydospore Test}

The purpose of this test is to differentiate Candida species from albicans and non-albicans, as well as to a large extent, to detect non-albicans such as C. glabra, C. tropicalis, and C. krusei. Using a chromogenic medium and the fungal samples after purification, it is cultured on this medium. They are incubated at $30^{\circ} \mathrm{C}$ for 48 hours and then the type of fungal is confirmed based on the colony color. Similar to the germ tube formation, this feature is also specific to C. albicans. In this study, the yeasts were cultured through a corn meal agar medium and then were kept at $25^{\circ} \mathrm{C}$ for a week. In addition to chlamydospore, C. albicans forms pseudohyphae in this medium (33). CHROMagar Test 
Chromogenic Candida agar aims to differentiate Candida species based on the color which is produced on the media. In this study, fungal species were purified, cultured, and incubated at $30^{\circ} \mathrm{C}$ for 48 hours using a chromogenic medium. Then, eight colors were observed as green, light green, green with a blue border, bluish green, purple, blue, white, and white with blue spots. The first four colors were all categorized as green and were interpreted as C. albicans $(33,34)$.

Some materials that were included in the Sabouraud dextrose agar, Corn Meal Agar, disc diffusion clotrimazol, fluconazole, and nystatin were purchased from Himedia in India.

The inclusion criteria included being married, not having vaginal bleeding and chronic diseases, not using herbal and chemical drugs for the treatment of genital infection in the recent 2 weeks, as well as not performing vaginal douching, creams, or suppositories within the past 48 hours. The exclusion criteria were the negative fungal culture in Sabouraud dextrose agar medium and negative direct test in the lam staining.

In order to collect the samples, the patients were required to lie in lithotomic position and the samples were gathered from vaginal discharges using a speculum and sterile swab. For each patient, 2 swabs were used for preparing direct smear and culturing the sample in Sabouraud dextrose agar medium under a sterile condition. It should be mentioned that the number, date, and other characteristics of the samples were recorded on the plates containing the culture media. The plates were daily transferred to the mycology laboratory of the school of medicine and kept in the incubator at $30^{\circ} \mathrm{C}$ for $24-48$ hours. Next, the colonies were passaged for purification and were then kept in sterile distilled water at $-20^{\circ} \mathrm{C}$ for further experiments.

In order to prepare T. polium smoke, the plant from the Asteraceae family was identified and approved by a specialist in medicinal plants. Then, it was transferred to the pharmacognosy laboratory of the school of pharmacy. In the laboratory, first, $1000 \mathrm{~g}$ of the plant was powdered using a grinder. Afterward, it was heated in a device with a $1000 \mathrm{cc}$ balloon at the bottom and a converter on the top for one hour. In this way, the plant was turned into smoke, moved upwards, collected in the converter, and changed into a liquid. In addition, the solvent of the liquid was extracted using nitrogen and the pure smoke was prepared to perform drug sensitivity tests. At this stage, T. polium smoke product was prepared in pure concentration and a soluble form was created in the polar solutes. Further, disks were provided containing different concentrations of $T$. polium smoke product (i.e., 10, 20, 30, 60, 90, 120, 180, 210, and 240 lambdas). The researchers also applied nystatin disks containing 100 unit/disk medicine made by Himedia Company, India. The concentration of the fungal suspension was determined based on McFarland 0.5 turbidity standard and was cultured on Sabouraud dextrose agar medium by a sterile swab. The aforementioned disks were located on the medium for 48 hours and the inhibition zones around the disks (representing the antifungal effects of the drugs) were evaluated accordingly. In addition, the effect of $T$. polium smoke product was assessed on five standard Candida strains including C. albicans, C. glabrata, C. tropicalis, C. krusei, and C. parapsilosis, as well as 10 clinical Candida species which were isolated from VVC in the present study. Then, the antifungal effect of T. polium smoke product was determined according to the presence or absence of the inhibition zone around the disks.

At the end of the incubation stage, the diameters of the inhibition zones around the nystatin and T. polium disks were measured and compared as well. It should be noted that the sensitivity or resistance to the antifungal drugs was determined based on the measures mentioned in the brochure provided by the company manufacturing the disks (Himedia, India). Accordingly, the inhibition zones with $<15 \mathrm{~mm}$ diameters were considered resistant while those with $\geq 15 \mathrm{~mm}$ diameters were found to be sensitive to nystatin.

Based on the investigation of the antifungal effects of $T$. polium smoke product, no inhibition zones were formed in the disks containing $10,20,30,60$, and $90 \lambda$ medicine. However, the inhibition zone started to form around the disk containing $120 \lambda$ medicine, which indicates that the antifungal activities of T. polium smoke product began at this concentration. Although the inhibition zone was yet larger around the disks containing 180 and $210 \lambda$ medicine, they were not different from those which were created around $240 \lambda$ disk. Therefore, the amount of medicine increased no more, and $180 \lambda$ disks were considered as the minimum concentration preventing the fungal growth. Finally, the collected data were entered the SPSS statistical software (version 16) and analyzed using the chi-square test and descriptive statistics.

\section{Results}

According to the results of the present study, the mean age of the patients was $32 \pm 9.49$ years, and the highest proportion of infection was related to 26-35 years agegroup. Among the 450 patients with the clinical symptoms of VVC, 102 cases showed positive fungal colony cultures, and 2 fungal species grew in three cases. Thus, a total of 105 cases $(23.3 \%)$ were culture positive. Based on the germ tube test and chlamydospore test, $70.5 \%$ and $23.8 \%$ of the cases were C. albicans (Table 1). In CHROMagar test, $66.6 \%, 21.9 \%, 8.6 \%$, and $2.9 \%$ of the samples were also C. albicans, C. glabrata, and the other non-albicans, C. tropicalis, and C. krusei, respectively. Based on the diagnostic test for chlamydospore test, $54.3 \%, 21.9 \%$, and $23.8 \%$ of cases were reported as Candida albicans, yeast, and of Candida non-albicans as well.

The study results showed that $99 \%$ of the samples were sensitive to nystatin and a significant relationship was 
Table 1. Candidates Based on a Variety of Diagnostic Tests in Women With Vaginal Candiditis

\begin{tabular}{lccc}
\hline Tests & $\begin{array}{c}\text { Germ Tube } \\
\text { Test } \\
\text { No. (\%) }\end{array}$ & $\begin{array}{c}\text { Chlamydospore } \\
\text { Formation } \\
\text { No. (\%) }\end{array}$ & $\begin{array}{c}\text { CHROMagar } \\
\text { Candida } \text { Test } \\
\text { No. (\%) }\end{array}$ \\
\hline Albicans & $74(70.5)$ & $57(45.3)$ & $40(66.6)$ \\
Non-albicans & $31(29.5$ & $48(45.7)$ & $35(33.4)$ \\
\hline
\end{tabular}

observed between the antifungal drugs and the types of organisms $(P \leq 0.02)$. Moreover, all the 15 standard clinical samples were sensitive to T. polium smoke. (Table 2). The inhibition zone around 105 nystatin disks ranged from 13.5 to $37 \mathrm{~mm}$ with an average diameter of $21 \pm 2.98 \mathrm{~mm}$. Additionally, the inhibition zones were formed around all the nystatin disks and none of the samples showed 100\% resistance to this medicine (Table 3 ).

On the other hand, T. polium smoke product saturated with $180 \lambda$ medicine was used for five standard Candida strains including glabrata, tropicalis, krusei, parapsilosis, and albicans, as well as for 10 samples which were obtained from the study patients. According to the results, the mean diameter of the inhibition zone was $13 \mathrm{~mm}$.

\section{Discussion}

The results revealed that $66.6 \%$ of the species were $C$. albicans while $33.4 \%$ of them were non-albicans. In the study by Moreira et al, $63 \%$ of the patients with the clinical diagnosis of Candida showed positive cultures, $95 \%$ of which were related to C. albicans (35). In another study in India, $65.73 \%$ of the Candida cultures included nonalbicans species as well (36). In addition, Panahi et al found that $50.8 \%$ of the cases were positive among which, $68.8 \%$ were related to C. albicans (37). Based on the reports of Mirhendi et al, $66.5 \%$ of the cases were also C. albicans whereas the rest of them were Candida non-albicans (38).

Similarly, the results of the study by Mucci et al showed that the presence of C. albicans and C. dubliniensis and the absence of $C$. africana in pregnancy were significant so

Table 2. Distribution of the Susceptibility of Candida Species Isolated From the Vagina to the Drugs

\begin{tabular}{lcc}
\hline Drugs & $\begin{array}{c}\text { Nystatine } \\
\text { No. (\%) }\end{array}$ & $\begin{array}{c}\text { Teucrium polium Smoke Product } \\
\text { No. (\%) }\end{array}$ \\
\hline Sensitive & $104(99)$ & $15(100)$ \\
Resistance & $1(1)$ & $0(0)$ \\
$P$ value $^{\mathrm{a}}$ & 0.02 & 0.00 \\
\hline
\end{tabular}

${ }^{\mathrm{a}}$ Chi-square test. that about $80.7 \%$ (42 cases) of the samples had C. albicans, which was observed in $7.5 \%$ ( 3 cases) with other species of the genus. This is in line with the results of the present study regarding the prevalence of a candidate (39).

Richter et al concluded that azoles were less effective in non-albicans Candida species while nystatin was effective in $90 \%$ of the patients (7). Despite the increase in the incidence of non-albicans species, they demonstrate more resistance to azoles and their resultant infections are difficult to treat accordingly $(7,40)$. The higher prevalence of non-albicans vaginitis might be justified by an excessive increase in the utilization of tropical azoles in some countries since 1992 (7). Moreover, the high prevalence rate of $C$. albicans might be attributed to its higher capability in adherence to the vaginal epithelium (5).

In the present study, 2 fungal species were isolated from 3 cases. In the study by Richter et al (7), compound infections (mostly C. albicans and C. glabrata) were isolated from 27 out of 429 cases with VVC as well (5\%). However, Fan reported that more than one Candida species was responsible for VVC in only $0.02 \%$ of a total of 1070 patients (41).

The findings of the current study indicated that $T$. polium smoke had desirable effects on preventing the growth of C. albicans and non-albicans. Similarly, Qabaha demonstrated that the extracts of 5 medicinal plants (i.e., grape seed, $R$. officinalis, P. guajava, P. granatum peel, and T. polium) had antimicrobial effects on three Gram-positive microorganisms (i.e., S. aureus, B. subtilis, and $M$. luteus), 3 gram-negative cases (i.e., E. coli, $P$. aureuginosa, and $K$. pneumonia), and finally, 2 fungal species (C. albicans and A. niger) (26). In addition, the other researcher suggested that these medicines could be used as natural fungistatic, fungicidal, bactericidal, bacteriostatic, and antioxidant components in natural preservatives, as well as pharmaceutical products (30). Furthermore, the findings of another study performed in Iran demonstrated that T. polium could be an appropriate choice for the treatment of ulcerative colitis and Crohn's disease (42). Tepe et al also examined the effect of $T$. polium and T. chamaedrys on trophozoites and cysts in vitro and revealed no viable trophozoites at $32 \mathrm{mg} / \mathrm{mL}$ concentration within 48 hours (43).

In the study by Kremer et al. on chemical and other compounds, especially Teucrium arduini L. (Lamiaceae), the results represented that the plant had the properties of antimicrobial activity on Staphylococcus aureus, C.

Table 3. Relationship Between VVC Species, the Germ Tube Test and Chlamydospore Test

\begin{tabular}{lccccc}
\hline Chlamydospor & Positive & Yeast & Pseudohyphae & Negative & $\boldsymbol{P}$ Value \\
\hline Germ tube & No. $(\%)$ & No. $(\%)$ & No. $(\%)$ & No. (\%) \\
Albicans & $58(55.2)$ & $5(4.8)$ & $11(10.4)$ & $0(0)$ & $4(3.8)$ \\
Non-albicans & $0(0)$ & $18(17.1)$ & $9(8.6)$ & 20 & 4 \\
Total & 58 & 23 & 105 & 4 \\
\hline
\end{tabular}

VVV: Vaginal candidiasis infection; *Chi-square test. 
albicans, and several other microorganisms (44).

Likewise, Taheri et al. reported that different extracts of $A$. sieberi affected C. albicans and prevented its growth (21). Thus, they suggested more examinations in order to survey the composition of this plant and more extensive use in human domains. The results corroborate with the findings of the current study. In both studies, the herbal drug stopped the growth of Candida albicans. Further, Gholampour-Azizi I et al investigated the in vitro antifungal activity of Cucumis melo in Candida albicans by utilizing the disc and well diffusion and found that C. melo extrusion has a therapeutic and specific antifungal potential against $C$. albicans (20). In another study, Nadimi et al indicated that Teucrium polium extract has a significant effect on C. albicans and highlighted the need for further research in order to identify the effects of this plant in the treatment of Candida infections (45).

Therefore, Bahramikia and Yazdanparast reviewed over 100 articles evaluating the effects of pharmacology and toxicology extracts and the compounds isolated from T. polium, which were published during 1970-2011. They reported that these compounds have a wide range of pharmacological effects including antioxidant, anticancer, anti-inflammatory, blood sugar, protect the liver, lipid-lowering, anti-bacterial and antifungal activities (22).

Considering the comparison of the effects of T. polium and nystatin, the utilized disks were not of a similar type. Nystatin was prepared based on the unit while T. polium was provided based on $\lambda$. Thus, a quantitative comparison was impossible. Qualitatively, however, T. polium showed relative antifungal sensitivity as compared to nystatin. The relative effect of $T$. polium smoke might be because the components and derivatives of this plant should be separated in order to determine its active ingredient. Furthermore, due to the lack of studies on T. polium smoke in Iran, the results could not be compared to those of the other studies. On the other hand, such studies investigated other forms of T. polium such as essence, oil, and decoction, which also restricts the comparison.

Financial restrictions were considered as one of the limitations of this study. In addition, the researchers faced with the lack of laboratory experts when providing $T$. polium smoke, and therefore, extended the duration of the projects.

Suggestions for Further Research

1. Conducting research on human subjects rather than the medium;

2. Using a liquid medium instead of the disk drug for determining the sensitivity tests, particularly in the case of fluconazole;

3. Preparing T. polium powder instead of a liquid for assessing the effect of the antifungal plant;

4. Preparing aqueous and alcoholic extracts instead of $T$. polium smoke for antifungal effect;

5. Analyzing T. polium smoke and more precise identification of the antifungal compounds of the plant.

\section{Conclusions}

Overall, the findings of this study showed the antifungal effects of T. polium on the species isolated from 10 clinical specimens of Candida vaginitis obtained in the present study, as well as on five standard strains of Candida including C. albicans, C. glabrata, C. tropicalis, C. krusei, and $C$. parapsilosis which had a relatively good sensitivity as compared to the chemical drug nystatin. To the best of our knowledge, this is the first research that has been conducted in this area in Iran and after extraction of the compounds of this plant, stronger antifungal effects might yet be achieved in future studies.

\section{Conflict of Interests}

The authors declared no potential conflicts of interest with respect to the research, authorship, and/or publication of this article.

\section{Ethical Issues}

This research project was approved by the Ethics Committee of Shiraz University of Medical Sciences and written informed consent was obtained from all the participants.

\section{Financial Support}

This article is a part of the thesis submitted by Batol Bonyadpour (thesis number: 3853). This study was financially supported by the Research Vice-chancellor of Shiraz University of Medical Sciences.

\section{Acknowledgments}

Hereby, the authors would like to thank the deans of the gynecology clinics of Shahid Motahari, Zeinabiyeh, and Hor-e-Riahi Hospitals affiliated to Shiraz University of Medical Sciences. Special thanks also go to the Center for Development of Clinical Research of Nemazee Hospital and Dr. Nasrin Shokrpour for editorial assistance. The researchers appreciate the Research and Technology Department of Shiraz University of Medical Sciences for financial support..

\section{References}

1. Apalata T, Longo-Mbenza B, Sturm A, Carr W, Moodley P. Factors associated with symptomatic vulvovaginal candidiasis: a study among women attending a primary healthcare clinic in Kwazulu-Natal, South Africa. Ann Med Health Sci Res. 2014;4(3):410-416. doi:10.4103/21419248.133470

2. Apalata T, Carr WH, Sturm WA, Longo-Mbenza B, Moodley P. Determinants of symptomatic vulvovaginal candidiasis among human immunodeficiency virus type 1 infected women in rural KwaZulu-Natal, South Africa. Infect Dis Obstet Gynecol. 2014;2014:387070. doi:10.1155/2014/387070 
3. Pfaller MA, Diekema DJ. Epidemiology of invasive candidiasis: a persistent public health problem. Clin Microbiol Rev. 2007;20(1):133-163. doi:10.1128/ cmr.00029-06

4. Akbarzadeh M, Bonyadpoure B, Pacshir K, Mohagheghzadeh A. Causes and clinical symptoms of vaginal candidiasis in patients referring to selective clinics of Shiraz University of Medical Sciences (2009). Journal of Arak University of Medical Sciences. 2010;13(3):12-20. [Persian].

5. Grigoriou O, Baka S, Makrakis E, Hassiakos D, Kapparos G, Kouskouni E. Prevalence of clinical vaginal candidiasis in a university hospital and possible risk factors. Eur J Obstet Gynecol Reprod Biol. 2006;126(1):121-125. doi:10.1016/j. ejogrb.2005.09.015

6. Mahmoudi Rad M, Zafarghandi S, Abbasabadi B, Tavallaee M. The epidemiology of Candida species associated with vulvovaginal candidiasis in an Iranian patient population. Eur J Obstet Gynecol Reprod Biol. 2011;155(2):199-203. doi:10.1016/j.ejogrb.2010.11.022

7. Richter SS, Galask RP, Messer SA, Hollis RJ, Diekema DJ, Pfaller MA. Antifungal susceptibilities of Candida species causing vulvovaginitis and epidemiology of recurrent cases. J Clin Microbiol. 2005;43(5):2155-2162. doi:10.1128/ jcm.43.5.2155-2162.2005

8. Ehrström SM, Kornfeld D, Thuresson J, Rylander E. Signs of chronic stress in women with recurrent Candida vulvovaginitis. Am J Obstet Gynecol. 2005;193(4):13761381. doi:10.1016/j.ajog.2005.03.068

9. Dovnik A, Golle A, Novak D, Arko D, Takac I. Treatment of vulvovaginal candidiasis: a review of the literature. Acta Dermatovenerol Alp Pannonica Adriat. 2015;24(1):5-7.

10. Tseng TY, Chen TC, Ho CM, et al. Clinical features, antifungal susceptibility, and outcome of Candida guilliermondii fungemia: An experience in a tertiary hospital in mid-Taiwan. J Microbiol Immunol Infect. 2018;51(4):552-558. doi:10.1016/j.jmii.2016.08.015

11. Choukri F, Benderdouche $M$, Sednaoui P. In vitro susceptibility profile of 200 recent clinical isolates of Candida spp. to topical antifungal treatments of vulvovaginal candidiasis, the imidazoles and nystatin agents. J Mycol Med. 2014;24(4):303-307. doi:10.1016/j. mycmed.2014.05.001

12. Martins HP, da Silva MC, Paiva LC, Svidzinski TI, Consolaro ME. Efficacy of fluconazole and nystatin in the treatment of vaginal Candida species. Acta Derm Venereol. 2012;92(1):78-82. doi:10.2340/00015555-1194

13. Noumi E, Snoussi M, Hajlaoui H, Valentin E, Bakhrouf A. Antifungal properties of Salvadora persica and Juglans regia L. extracts against oral Candida strains. Eur J Clin Microbiol Infect Dis. 2010;29(1):81-88. doi:10.1007/ s10096-009-0824-3

14. Akbarzadeh M, Moshfeghy Z, Dehghani M, Emamghoreishi M, Tavakoli P, Zare N. Comparison of the effect of Melissa officinalis capsule and care educational programs on the intensity of physical, mental and social symptoms of premenstrual syndrome in high school female students. Int J Womens Health Reprod Sci. 2018;6(1):18-26. doi:10.15296/ ijwhr.2018.05

15. Zeinalzadeh S, Akbarzadeh M, Faridi P, Mohagheghzadeh
AA, Sayadi M. Effect of sildenafil citrate on women affected by sexual dysfunction referred to health clinics. Family Medicine \& Primary Care Review. 2017;19(2):167-172. doi:10.5114/fmpcr.2017.67873

16. Heydari N, Dehghani M, Emamghoreishi M, Akbarzadeh M. Effect of Melissa officinalis capsule on the mental health of female adolescents with premenstrual syndrome: a clinical trial study. Int J Adolesc Med Health. 2018. doi:10.1515/ijamh-2017-0015

17. Akbarzadeh M, Zeinalzadeh S, Zolghadri J, Mohagheghzadeh A, Faridi P, Sayadi M. Comparison of Elaeagnus angustifolia extract and sildenafil citrate on female orgasmic disorders: a randomized clinical trial. J Reprod Infertil. 2014;15(4):190-198.

18. Akbarzadeh M, Dehghani M, Moshfeghy Z, Emamghoreishi M, Tavakoli P, Zare N. Effect of Melissa officinalis capsule on the intensity of premenstrual syndrome symptoms in high school girl students. Nurs Midwifery Stud. 2015;4(2):e27001. doi:10.17795/nmsjournal27001

19. Heidary M, Yazdanpanahi Z, Dabbaghmanesh MH, Parsanezhad ME, Emamghoreishi M, Akbarzadeh M. Effect of chamomile capsule on lipid- and hormonalrelated parameters among women of reproductive age with polycystic ovary syndrome. J Res Med Sci. 2018;23:33. doi:10.4103/jrms.JRMS_90_17

20. Gholampour-Azizi I, Rouhi S, Yahyayi F. In vitro antifungal activity of Cucumis melo on Candida albicans. Zahedan J Res Med Sci. 2015;17(7):35-39. doi:10.17795/zjrms1019

21. Taheri AA, Gholampourazizi I, Hashemi Karoui M, Farhadi L, Servatyari K, Rouhi S. Inhibitory effect of aquatic and alcoholic extracts of Artemisia sieberi on growth of Candida albicans: an in vitro study. Qom Univ Med Sci J. 2018;12(6):39-47. doi:10.29252/qums.12.6.39

22. Bahramikia S, Yazdanparast R. Phytochemistry and medicinal properties of Teucrium polium L. (Lamiaceae). Phytother Res. 2012;26(11):1581-1593. doi:10.1002/ ptr.4617

23. Naghibi F, Mosaddegh M, Mohammadi Motamed M, Ghorbani A. Labiatae family in folk medicine in Iran: from ethnobotany to pharmacology. Iran J Pharm Res. 2010;4(2):63-79. doi:10.22037/ijpr.2010.619

24. Zargari A. Medicinal plants. 6th ed. Tehran: Tehran University Publications; 1997.

25. Khoshnood-Mansoorkhani MJ, Moein MR, Oveisi N. Anticonvulsant activity of Teucrium polium Against Seizure Induced by PTZ and MES in Mice. Iran J Pharm Res. 2010;9(4):395-401.

26. Qabaha KI. Antimicrobial and free radical scavenging activities of five Palestinian medicinal plants. Afr J Tradit Complement Altern Med. 2013;10(4):101-108. doi:10.4314/ ajtcam.v10i4.17

27. Zhang Z, Chang Q, Zhu M, Huang Y, Ho WK, Chen Z. Characterization of antioxidants present in hawthorn fruits. J Nutr Biochem. 2001;12(3):144-152.

28. Alizadeh AM, Sohanaki H, Khaniki M, Mohaghgheghi MA, Ghmami G, Mosavi M. The effect of teucrium polium honey on the wound healing and tensile strength in rat. Iran J Basic Med Sci. 2011;14(6):499-505.

29. Capasso F, Cerri R, Morrica P, Senatore F. Chemical composition and anti-inflammatory activity of an alcoholic 
extract of Teucrium polium L. Boll Soc Ital Biol Sper. 1983;59(11):1639-1643.

30. Mitić V, Jovanović O, Stankov-Jovanović V, Zlatkovic B, Stojanovic G. Analysis of the essential oil of Teucrium polium ssp. capitatum from the Balkan Peninsula. Nat Prod Commun. 2012;7(1):83-86.

31. Mukasa KJ, Herbert I, Daniel A, Sserunkuma KL, Joel B, Frederick B. Antifungal susceptibility patterns of vulvovaginal Candida species among women attending antenatal clinic at Mbarara Regional Referral Hospital, South Western Uganda. Br Microbiol Res J. 2015;5(4):322331. doi:10.9734/bmrj/2015/13804

32. Bonyadpour B, Akbarzdeh $\mathrm{M}$, Mohagheghzadeh AA. Characterization of Candida Species Isolated from cases of vulvovaginitis in women referring to selected gynecological clinics. J Midwifery Reproductive Health. 2016;4(4):741747. doi:10.22038/jmrh.2016.7562

33. Woodford N, Afzal-Shah M, Warner M, Livermore DM. In vitro activity of retapamulin against Staphylococcus aureus isolates resistant to fusidic acid and mupirocin. J Antimicrob Chemother. 2008;62(4):766-768. doi:10.1093/ $\mathrm{jac} / \mathrm{dkn} 266$

34. Foongladda S, Haouharn P, Sakulmaiwatana P, Chaiprasert A. Comparative evaluation of Candi Select test and conventional methods for identification of Candida albicans in routine clinical isolates. Mycoses. 2002;45(34):75-78. doi:10.1046/j.1439-0507.2002.00728.x

35. Moreira D, Paula CR. Vulvovaginal candidiasis. Int J Gynecol Obstet. 2006;92(3):266-267. doi:10.1016/j. ijgo.2005.12.007

36. Kumari V, Banerjee T, Kumar P, Pandey S, Tilak R. Emergence of non-albicans Candida among candidal vulvovaginitis cases and study of their potential virulence factors, from a tertiary care center, North India. Indian J Pathol Microbiol. 2013;56(2):144-147. doi:10.4103/03774929.118703

37. Nozhat F, Zarei M. Association between clinical symptoms and laboratory findings in the diagnosis of Candida vaginitis. Feyz Journal. 2016;20(2):173-179. [Persian].

38. Mirhendi SH, Makimura K, Shidfar MR, Hossienpour L. Identification and frequency of Candida patient isolates by CHROMagar Candida method. Avicenna Journal of Clinical Medicine. 2007;13(4):11-15. [Persian].

39. Mucci MJ, Cuestas ML, Landanburu MF, Mujica MT. Prevalence of Candida albicans, Candida dubliniensis and Candida africana in pregnant women suffering from vulvovaginal candidiasis in Argentina. Rev Iberoam Micol. 2017;34(2):72-76. doi:10.1016/j.riam.2016.09.001

40. Nucci $M$, Silveira MI, Spector $N$, et al. Fungemia in cancer patients in Brazil: predominance of nonalbicans species. Mycopathologia. 1998;141(2):65-68. doi:10.1023/a:1006951619245

41. Fan SR, Liu XP, Li JW. Clinical characteristics of vulvovaginal candidiasis and antifungal susceptibilities of Candida species isolates among patients in southern China from 2003 to 2006. J Obstet Gynaecol Res. 2008;34(4):561566. doi:10.1111/j.1447-0756.2008.00817.x

42. Mehrabani D, Bahrami F, Hosseini SV, et al. The healing effect of Teucrium polium in acetic acid-induced ulcerative colitis in the dog as an animal model. Middle East J Dig Dis. 2012;4(1):40-47.

43. Tepe B, Malatyali E, Degerli S, Berk S. In vitro amoebicidal activities of Teucrium polium and T. chamaedrys on Acanthamoeba castellanii trophozoites and cysts. Parasitol Res. 2012;110(5):1773-1778. doi:10.1007/s00436-0112698-4

44. Kremer D, Kosir IJ, Kosalec I, et al. Investigation of chemical compounds, antioxidant and antimicrobial properties of Teucrium arduini L. (lamiaceae). Curr Drug Targets. 2013;14(9):1006-1014. doi:10.2174/1389450111314090009

45. Nadimi M, Zia M, Madani M. The effect of aqueous and ethanolic extracts of Teucrium polium on Candida albicans and two species of Malassezia. Zahedan J Res Med Sci. 2013;15(8):34-38.

(c) 2019 The Author (s); This is an open-access article distributed under the terms of the Creative Commons Attribution License (http://creativecommons.org/licenses/by/4.0), which permits unrestricted use, distribution, and reproduction in any medium, provided the original work is properly cited. 